\title{
Soft tissue changes inconclusive in Class II division 1 patients treated with Activator and Bionator appliances
}

\author{
Does the cephalometric facial soft tissue change after the use of the Activator \\ and Bionator appliances in Class II division 1 patients?
}

\begin{abstract}
Flores-Mir C, Major PW. A systematic review of cephalometric facial soft tissue changes with the Activator and Bionator appliances in Class II division 1 subjects. Eur J Orthod 2006; 28:586-593
\end{abstract}

\begin{abstract}
Data sources Medline, Medline In-Process and other non-indexed citations, Lilacs, PubMed, Embase, Web of Science and all evidence-based medicine reviews were searched. The reference lists of the retrieved articles were also searched by hand for possible missing articles. Authors were contacted to obtain additional information if necessary.

Study selection For inclusion, an article had to satisfy the following criteria: they should be clinical trials with a comparable untreated control group; facial soft tissue changes were evaluated through lateral cephalograms; Activator and/or Bionator functional appliances were used to correct Class II division 1 malocclusions; no syndromic or medically compromised patients were included; none were individual case-reports or series of cases; there was no surgical intervention; and only a removable functional appliance was used.
\end{abstract}

Data extraction and synthesis Screening of eligible studies was independently made by both authors and their results were compared: discrepancies were settled through discussion. In the case of the Lilacs database, evaluation was by one author alone because of language limitation. Eligible studies were independently evaluated by both authors using a methodological scoring process which was developed to identify which selected studies would be most valuable.

Results The search identified 30 articles, of which 11 met the inclusion criteria. Only one of these was a randomised controlled trial. Five studies evaluated the soft tissue changes after use of an Activator appliance. No changes in the naso-labial and labio-mental angles were observed, but a mild protrusion of menton was reported. Neither the tip nor the base of the nose underwent any change. Contradictory results were found regarding the position of the upper lip, the lower lip and menton. Contradictory changes in upper lip thickness and length were also reported, but no changes in the lower lip or soft tissue menton were noted.

Six studies evaluated the soft tissue changes using a Bionator. Contradictory results were reported for the facial angles. No studies reported a significant naso-labial angle change. Total face height and lower face thirds were augmented. Contradictory results were found for the antero-posterior position of the upper lip, lower lip and soft-tissue pogonion. A vertical increase was reported for upper lip, lower lip and soft tissue menton measurements.

Conclusions Based on the available evidence, a significant amount of controversy regarding the soft tissue changes produced by the Activator and the Bionator exists. Soft tissue changes that were reported as being statistically significant were of questionable clinical significance.

Address for correspondence: Dr Carlos Flores-Mir, Faculty of Medicine and Dentistry, Room 4051A, Dentistry/ Pharmacy Centre, University of Alberta, Edmonton, Alberta, Canada T6G 2N8. E-mail: carlosflores@ualberta.ca

\section{Commentary}

This systematic review intended to explore evidence from the literature regarding cephalometric soft tissue changes following functional appliance treatment. The methodology was elaborate. The selected articles were critically evaluated, and the reasons for inclusion and exclusion were clearly described. The outcome of this review in terms of the number of included studies and consistency of their results, however, did not meet expectations because of the remarkable controversy within the literature. Based on the available evidence, clinicians cannot promise their growing patients a more attractive facial profile with functional appliance treatment.

It is interesting to note that, of the 11 articles included, five were not published in English (three were written in Portuguese, one in Turkish, and one was not clear because no abstract was available); four were not indexed in PubMed/Medline; and only five were published in core orthodontic journals (three in European Journal of Orthodontics, one in Angle and one in the American Journal of Orthodontics and Dentofacial Orthopedics). This presents an embarrassing dilemma — why were many good studies not published in English core dentistry journals? And how can we, as readers limited by the language and resource, re-evaluate these studies? A comprehensive database search in all possible languages is necessary for good systematic reviews, but it does involve considerable work. For the benefit of readers and authors alike, publishing data in journals with a wide readership and an easier accessibility should be encouraged.

Of all the types of functional appliances, Activator and Bionator were selected for the study because they are most commonly used. This may not necessarily mean, however, that more or better publications are available describing these two devices. In fact, only five studies for Activator and six for Bionator fulfilled the inclusion criteria, and neither provided sound evidence. It would be interesting to look into other types of appliances, eg, the toothborne active type, which hopefully is ongoing. Regarding the evaluation of facial attractiveness, it is slightly unconvincing that only one study ${ }^{1}$ of Activator and Frankel was the basis of the conclusion that there were statistically significant soft tissue changes after the use of the Activator or Bionator: this has a questionable clinical significance from layperson's perspective. Three-dimensional analysis of soft tissue changes was proposed as a better approach for future studies but the reasoning could be more clear. Can we attribute the lack of soft tissue changes to the drawbacks of two-dimensional measurement methods rather than the quality of the studies? If not, can we expect that a three-dimensional approach would yield different results?

\section{Yijin Ren \\ Department of Orthodontics, University Medical Centre Groningen, Groningen, The Netherlands}

1. O'Neill K, Harkness M, Knight R 2000 Ratings of profi le attractiveness after functional appliance treatment. Am J Orthod Dentofacial Orthop.118 : 371-376; discussion 377.

Evidence-Based Dentistry (2007) 8, 5-6. doi:10.1038/sj.ebd.6400495 